Archives de sciences sociales des religions

155 | juillet-septembre 2011

Le consensus des experts | Inattendus pèlerinages

\title{
L'«hétérotopie» des Sept Dormants en Bretagne
}

\section{Manoël Pénicaud}

\section{(2) OpenEdition}

Journals

Édition électronique

URL : http://journals.openedition.org/assr/23337

DOI : 10.4000/assr.23337

ISBN : 978-2-7132-2303-7

ISSN : $1777-5825$

Éditeur

Éditions de l'EHESS

Édition imprimée

Date de publication : 30 septembre 2011

Pagination : 131-148

ISSN : 0335-5985

Référence électronique

Manoël Pénicaud, «L'«hétérotopie» des Sept Dormants en Bretagne », Archives de sciences sociales des religions [En ligne], 155 | juillet-septembre 2011, mis en ligne le 10 novembre 2011, consulté le 01 mai 2019. URL : http://journals.openedition.org/assr/23337 ; DOI : 10.4000/assr.23337 


\section{Manoël Pénicaud}

\section{L'“ hétérotopie " des Sept Dormants en Bretagne}

Aussi curieux que cela puisse paraître, il existe un pèlerinage islamo-chrétien en Bretagne. Dédié aux Sept Dormants d'Éphèse - également connus en islam sous le nom d'Ahl al-Kahf ( "Gens de la Caverne ») -, il a été "inventé ", en 1954, par l'orientaliste Louis Massignon et est toujours en vigueur aujourd'hui. Chaque année, le quatrième week-end de juillet, des pèlerins chrétiens et musulmans se rejoignent au hameau des Sept-Saints, sur la lande bretonne du Trégor (Côtes d'Armor). L'originalité du sanctuaire est que la chapelle du XVIII ${ }^{\mathrm{e}}$ siècle est bâtie au-dessus d'un dolmen aménagé en crypte. Personne ne sait à quand remonte la christianisation de ce mégalithe, mais il évoque la grotte éphésienne (Turquie) où les Sept Dormants se seraient réveillés de leur sommeil miraculeux, au $\mathrm{V}^{\mathrm{e}}$ siècle ${ }^{1}$.

Pendant deux jours, le hameau connaît une effervescence inhabituelle et voit affluer plusieurs centaines de pèlerins. Mais force est de constater que la grande majorité est catholique, tandis que les musulmans sont très peu nombreux. Il faut ajouter par ailleurs des fréquentations secondaires: des agnostiques, quelques touristes, des adeptes du néo-celtisme, des new agers et quelques agnostiques épris de dialogue dit « interculturel».

La journée du samedi commence par des rencontres interreligieuses (colloque, débat, activités culturelles) dans la salle des fêtes de Vieux-Marché. À la tombée de la nuit, une messe est dite à la chapelle des Sept-Saints; elle est suivie d'une procession avec croix et bannières, jusqu'au traditionnel Tantad (feu de joie) allumé par le "pardonneur " ${ }^{2}$. La nuit se termine en Fez Noz aux sons des

1. Le mythe fondateur des Sept Dormants raconte comment sept jeunes Éphésiens du $\mathrm{III}^{\mathrm{e}}$ siècle refusent de renier leur foi chrétienne devant l'empereur Dèce et se réfugient dans une caverne proche de la ville. Les découvrant endormis, le souverain les fait emmurer vivants. Or, ils se réveillent 198 ans plus tard (la durée varie selon les versions, 372 dans la Légende Dorée), sous le règne de l'empereur chrétien Théodose II, avec l'impression de n'avoir dormi qu'une seule nuit. Le miracle vient confirmer le dogme de la résurrection des corps et se répand dans toute la chrétienté, mais aussi au-delà, puisqu'on le retrouve dans la sourate XVIII du Coran, où ces «Gens de la Caverne "sont considérés comme les seuls témoins anticipés de la résurrection. Ainsi, le mythe a essaimé aussi bien en terre chrétienne qu'en terre d'islam.

2. Le «pardonneur» est le prêtre qui préside le pardon. Notons qu'il a en ce lieu toujours un lien avec le monde arabo-musulman. 
binious et des cornemuses. Le dimanche matin, après la grand-messe catholique, environ deux cents personnes se rendent à la fontaine à sept trous, où la sourate XVIII est psalmodiée par un imam entouré d'une poignée de musulmans, puis traduite en français pour l'assistance. Un repas collectif (méchoui) est ensuite proposé dans un pré pour quatre cents convives ; enfin, les festivités reprennent tandis que quelques pèlerins se regroupent dans la chapelle pour un nouveau temps de dialogue islamo-chrétien organisé par le prêtre de la paroisse Notre Dame de Plouaret-Trégor.

Cet article se propose de retracer les grandes lignes de l'histoire du pèlerinage et d'en saisir à la fois les mutations et les enjeux révélés par son ethnographie. Nous allons donc suivre de l'intérieur la «fabrication » du pèlerinage, de même que l'émergence d'une « hétérotopie » islamo-chrétienne, ou plutôt celle de Louis Massignon. Enfin, nous questionnerons la pertinence de l' "islamo-christianité » d'un phénomène aussi complexe qu'inattendu.

D'un point de vue méthodologique, cette monographie est le fruit d'un croisement entre histoire et anthropologie. Ainsi, adopterons-nous une posture tantôt diachronique, tantôt synchronique. Sur le plan historique, cette recherche s'appuie en grande partie sur les archives inédites de Louis Massignon mises à notre disposition par sa famille ${ }^{3}$. Cette précieuse documentation (correspondances, coupures de presse, notes de travail, photographies, etc.) permet de suivre jour après jour la création du pèlerinage. Sur le plan anthropologique, les différentes ethnographies effectuées sur le terrain entre 2004 et 2009 abordent quant à elles la réalité contemporaine du phénomène.

Suivant ce découpage temporel, cet article se divise en deux parties, la première s'attache à la fondation du pèlerinage islamo-chrétien, et la seconde à la situation actuelle qui n'est pas exempte d'ambiguïtés.

\section{L'“ invention " d'un pèlerinage hétérotopique par Louis Massignon}

Tout pèlerinage naît apparemment d'un événement fondateur, que ce soit un miracle, une apparition ou la présence d'un saint personnage, mais il y a toujours, en filigrane, une figure ou une institution qui le développe concrètement. Ici, c'est bien Louis Massignon qui en est à l'origine, d'où la place de premier plan qu'il occupe dans cet article.

Parfois considéré comme « le dernier orientaliste », Louis Massignon (18831962 ) est l'un des pères de l'islamologie française. Titulaire de la chaire de sociographie musulmane au Collège de France, de 1926 à 1954, il a consacré sa vie à l'étude du monde arabo-musulman et de l'islam à travers lequel il a recouvré

3. Nous remercions vivement la famille Massignon pour la mise à disposition de ces archives inédites, dont quelques extraits sont cités dans cet article. 
sa foi catholique en 1908. Car il ne se cachait pas d'être croyant, si bien qu'à sa mort, on dit de lui qu'il fut « le plus grand musulman parmi les chrétiens et le plus grand chrétien parmi les musulmans ${ }^{4}$ ». Ainsi, est-il un pionnier du dialogue islamo-chrétien en France.

Sur un plan plus politique et social, Louis Massignon est un «intellectuel catholique " qui s'est engagé pour de multiples causes, dont la reconnaissance de l'islam, la non-violence et les décolonisations (à la fin de sa vie). Mais le personnage a souvent fait l'objet de controverses, si bien qu'il fut par exemple soupçonné d'espionnage sous la III ${ }^{\mathrm{e}}$ République. En fait, la vie de cet homme pluriel était relativement méconnue, jusqu'à ce qu'il connaisse, depuis peu, une certaine réhabilitation scientifique (Massignon, 2009a).

Nous pouvons toutefois affirmer que sa complexité réside dans sa double vocation de savant et de croyant (et notamment de mystique). Ajoutons qu'il a par ailleurs été secrètement ordonné prêtre dans le rite catholique melchite, en 1950, par un indult du pape Pie XII. La création du pèlerinage des Sept Dormants est donc avant tout l'œuvre d'un croyant et moins d'un universitaire. Ainsi, plus que le savant académique, nous avons découvert une facette méconnue du personnage : "l'entrepreneur » du religieux. À travers cette notion héritée de la vision wébérienne du champ religieux, nous voulons insister sur le caractère de «fabrication " du pèlerinage.

Pourquoi les Sept Dormants ? Louis Massignon voit en eux un trait d'union majeur entre christianisme et islam. Pour lui, c'est sur la base de l'attente commune de la résurrection que la réconciliation peut et doit se faire : son projet est donc éminemment eschatologique (Massignon, 2009b : 321-335) ${ }^{5}$. Ainsi, lorsqu'il parle de "pèlerinage de réconciliation " entre chrétiens et musulmans, il faut d'abord l'entendre dans un sens eschatologique. L'enjeu d'un tel rapprochement dépasse largement la Bretagne et consiste à préparer la réconciliation finale de l'humanité à Jérusalem. C'est ce que nous appelons "l'œcuménisme abrahamique » de Louis Massignon, qui inclut certes le judaïsme, mais en commençant par l'islam et le christianisme à travers les Sept Dormants.

Or, cet idéal - ou cette utopie - va se concrétiser et s'enraciner géographiquement. Dès lors, il devient une " hétérotopie », d'après le concept forgé par Michel Foucault, en 1967 (Foucault, 2001) ${ }^{6}$. Ce dernier désigne par là un " espace autre » ou une utopie réalisée et localisée dans l'espace. Selon nous, le pèlerinage

4. Hommage prononcé par Ibrahim Makdour le 20 décembre 1962, à l'académie arabe du Caire.

5. Signalons un article décisif sur le sens « apocalyptique» de la XVIII ${ }^{\mathrm{e}}$ sourate du Coran : Massignon (2009b).

6. Michel Foucault a donné cette conférence «Des espaces autres », le 14 mars 1967 au Cercle d'études architecturales, mais il n'en a autorisé la publication qu'en 1984 dans Architecture, Mouvement, Continuité, $\mathrm{n}^{\circ}$ 5, pp. 46-49. 
des Sept Dormants en Bretagne est donc la concrétisation de l'idéal massignonien de réconciliation abrahamique. Citons d'ailleurs l'orientaliste qui confie : «Ce but auquel j'ai donné ma vie ; le symbole le plus populaire que j'en ai trouvé, tant en Chrétienté qu'en Islam, est cette invocation par des pèlerins à ces ressuscités “anticipés”, Témoins avant-coureurs de la Justice, devant cette crypte-dolmen de la chapelle de Vieux-Marché ${ }^{7}$."

Pourquoi la Bretagne? C'est par sa fille ethnologue, Geneviève, que Louis Massignon découvre en 1952 le pardon des Sept-Saints de Vieux-Marché. Il est aussitôt frappé par certaines résonances entre le chant breton des Sept-Saints $\left(\right.$ Gwerz $\left.^{8}\right)$ et la sourate XXVIII du Coran. Très vite, il enclenche un processus de légitimation du lieu saint par ses recherches scientifiques (Massignon, 1992). Il s'attache à démontrer l'origine orientale de la vénération des Sept Dormants en Bretagne, là où d'autres n'y voyaient qu'un avatar de la dévotion aux sept évêques évangélisateurs de la Bretagne honorés lors du Tro Breizh ${ }^{9}$. L'homonymie des «Sept Saints » prêtait en effet à confusion. Il forge alors l'hypothèse de la route de l'étain, selon laquelle des marins grecs voyageant vers l'Angleterre auraient importé le culte des Dormants en Bretagne, mais là n'est pas notre sujet $^{10}$.

Retenons qu'il légitime son projet spirituel par ses recherches scientifiques. Il fait d'ailleurs de même au sujet d'autres sites des Sept Dormants qu'il recense à travers le monde et qui donne lieu à une série de publications majeures : "Les Sept Dormants d'Éphèse (Ahl Al-Kahf) en Islam et en Chrétienté. Recueil documentaire et iconographique "(Massignon, 1954-1963). Là encore, ces recherches encyclopédiques servent à étendre son projet "hétérotopique " en d'autres sites dédiés aux Sept Dormants (Maroc, Algérie, Rome, par exemple), (Pénicaud, 2011).

7. Lettre de Louis Massignon au ministre des Travaux publics, Robert Buron (24 juin 1962).

8. Pour une étude critique de la Gwerz et de l'interprétation faite par Massignon, voir : Laurent Donatien, 2004, "La Gwerz des Sept Saints Dormants ", Hopala! La Bretagne au monde, $\mathrm{n}^{\circ} 17$, pp. 32-42.

9. Le Tro Breizh (tour de la Bretagne, en breton) est le pèlerinage aux sept saints évêques évangélisateurs de la Bretagne : Corentin (Quimper), Pol Aurélien (Saint-Pol-de-Léon), Tugdual (Tréguier), Brieux (Saint-Brieux), Malo (Saint-Malo), Samson (Dol) et Patern (Vannes). Sur ce pèlerinage, voir : Brigitte Bleuzen, "Du Tro-Breiz à la Vallée des Mille Saints ", 2010, Archives de Sciences Sociales des Religions, 151, pp. 229-249; Jean Trévidy, 1897, "Les Sept Saints de Bretagne et leur pèlerinage ", Bulletin archéologique de l'Association Bretonne, Congrès de Rennes.

10. Remarquons toutefois que l'origine orientale de ce culte avait déjà été évoquée par Ernest Renan, interpelé par ce "culte venu d'Orient dans un cadre de préhistoire celtique ", avant de le faire remonter au haut Moyen Âge : "dès le VII siècle, une "acculturation" celtique de la légende syriaque des VII Dormants », Renan Ernest, 1878, « La légende des Sept Dormants en Basse Bretagne ", in Gaidoz H. et Rolland E., (dir.), Mélusine, Paris, col. 204-205. 


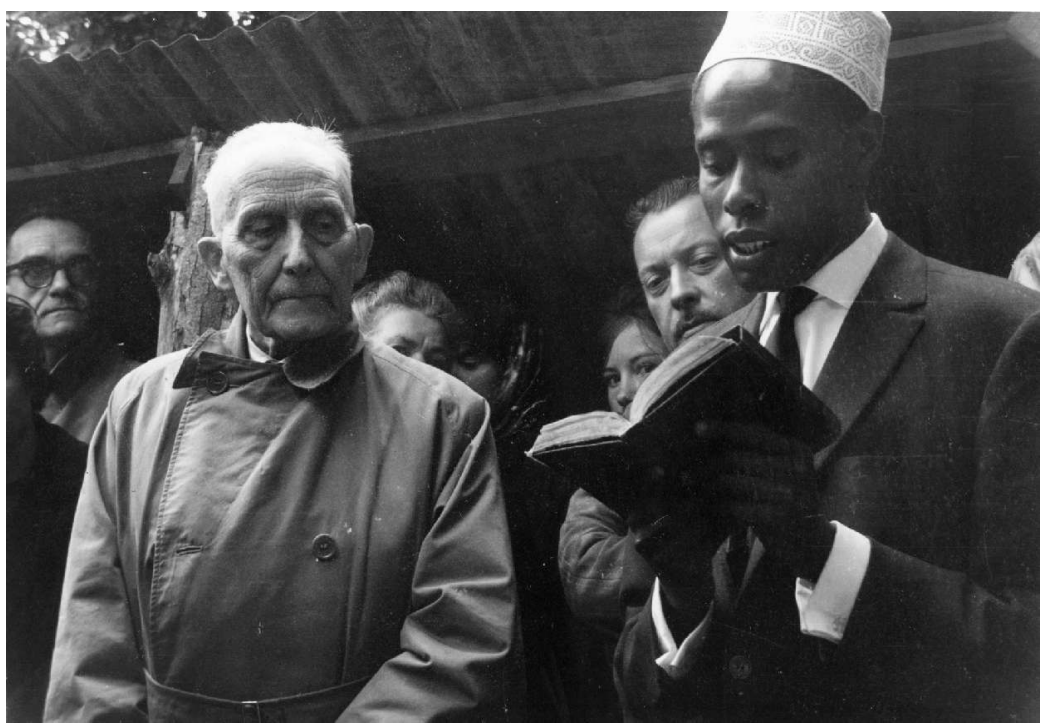

Louis Massignon, pendant son dernier pèlerinage, et Mohamed Taki

Le premier pèlerinage islamo-chrétien est organisé en 1954 pour " une paix sereine en Algérie ", et ce avant même que n'éclatent les événements de novembre. Plus que limitée la première année, la présence musulmane est renforcée l'année suivante par une délégation ouvrière de l'Amicale des Nord Africains Résidant en France (ANARF) venue de la région parisienne, et au sein de laquelle Massignon avait donné des cours d'alphabétisation dès 1929. Chaque année, entre une vingtaine et une quarantaine de pèlerins viendra en "pèlerinage organisé ", ce qui permet de lancer l'événement islamo-chrétien au sein du pardon catholique breton.

À la lecture de ses correspondances, Louis Massignon apparaît comme un véritable homme d'action et un fin stratège. Il supervise de près l'organisation du pèlerinage et œuvre quotidiennement à la réussite de l'entreprise. Dès le début, il s'assure le concours de soutiens locaux (le Recteur de Vieux-Marché, le maire et autres notables) et l'accord de l'évêché de Saint-Brieuc et Tréguier.

Parallèlement, il cherche assidûment des financements et mobilise ses différents réseaux de relations, notamment ecclésiastiques (archevêques, cardinaux, jusqu'au pape), diplomatiques (ambassadeurs) et politiques (ministres : Michel Debré, Georges Pompidou, jusqu'au Général De Gaulle ${ }^{11}$ ).

11. Lettre de M. Galichon, directeur du Cabinet de la Présidence, à Louis Massignon (30 juin 1962) : « C'est bien volontiers que le Général de Gaulle s'est plu à marquer l'intérêt qu'il porte à cette manifestation en faisant parvenir au compte postal de l'ANARF sa contribution personnelle, à laquelle il vous demande de bien vouloir conserver un caractère confidentiel. » 
De la même manière, il développe une véritable stratégie de communication et sollicite directement les rédacteurs en chef de certains journaux (Le Monde, La Vie, Ouest-France, Témoignage Chrétien, entre autres) qu'il connaît personnellement, afin de promouvoir le pèlerinage islamo-chrétien. D'année en année, l'événement est de plus en plus couvert, y compris par la radio puis par la télévision (RTF).

Sur place enfin, il est à l'origine du classement de la chapelle des Sept-Saints par les Monuments Historiques en 1956. Sa qualité de membre du Conseil des Musées Nationaux - où il siège avec André Malraux - a certainement contribué au bon traitement du dossier. En 1961, l'état de délabrement du bâtiment devient particulièrement inquiétant : "La toiture de la Chapelle ne supportera pas les pluies d'un nouvel hiver ${ }^{12}$ ", écrit-il au ministre Edmond Michelet, avant d'ajouter : "Et si elle s'effondre, comment faire fonctionner le Pèlerinage en 1962 ? Vous pouvez, d'un mot, la sauver, puisque les crédits sont prévus, et votés. » Le site faisait alors ses premiers pas sur le chemin de la patrimonialisation.

Pendant le pèlerinage proprement dit, les temps forts islamo-chrétiens sont la lecture de la Fatiha devant le dolmen, puis la participation des musulmans à la procession catholique vers le feu de joie (Tantad), en arborant une bannière où le début de l'Ave Maria est inscrit en arabe. Avec la venue de son ami Amadou Hampaté Bâ en 1961, "dont l'émotion et la conviction ont frappé le clergé local ${ }^{13}$ ", Louis Massignon convient qu'il faudra désormais inviter " un personnage canoniquement représentatif de l'Islam orthodoxe» pour contrebalancer la forte représentation ouvrière de la délégation musulmane ${ }^{14}$. C'est à partir de cette année que la fontaine à sept veines devient l'épicentre du pèlerinage islamochrétien et acquiert une nouvelle dimension sacrale. En inventant ce rituel de la psalmodie coranique, Massignon transforme la charge symbolique de cet espace où l'on venait pour les vertus curatives de l'eau de source.

Autre innovation : sur le plan liturgique chrétien, il institue une messe de rite melchite dite par un prêtre venu de Paris (Massignon ne voulait pas officier en public) en langue arabe. Il souhaite par là rapprocher les musulmans et les Bretons. Mais cette célébration, maintenue jusqu'en 1983, ne produit pas l'effet « œcuménique » espéré, puisque l'innovation engendre une concurrence entre messe orientale et messe latine.

$\mathrm{Au}$ niveau local, la réception du pèlerinage islamo-chrétien par les Bretons est mitigée, et ce d'autant plus que l'islam est totalement inconnu. Un témoin de l'époque raconte : "Ce n'était pas très bien accepté par les gens du quartier, surtout ceux qui avaient fait la guerre d'Algérie. Il y en avait quelques-uns qui

12. Lettre de Louis Massignon à Edmond Michelet, Garde des Sceaux (25 juillet 1961).

13. Lettre de Louis Massignon à Youakim Moubarac (24 juillet 1961).

14. Lettre de Louis Massignon au chanoine Du Cleuziou (7 août 1961). 
étaient assez mauvais même, mais il n'y a jamais eu de bagarre ni quoi que ce soit. Ils n'approuvaient pas. Ça s'est bien passé quand même. On ne pouvait pas désapprouver quelque chose qui veut rapprocher les gens et conduire à la paix, nous, chrétiens ${ }^{15}$.»

Le clergé local semble relativement dépassé par l'originalité de l'initiative que Massignon relaie dans les plus hautes sphères de l'Église romaine. Il ne cesse, en effet, de faire valoir que l'événement dépasse les prérogatives du diocèse et possède une dimension universelle. Aussi, sans s'y opposer, l'évêché suit le développement du phénomène avec prudence et circonspection.

Louis Massignon disparaît en 1962, juste avant le Concile de Vatican II dont il aura été à la fois un précurseur et un consultant ${ }^{16}$. Le pèlerinage traverse une période de succession difficile mais se maintient tant bien que mal. C'est finalement ses enfants (Daniel et Geneviève) et l'Association des Amis de Louis Massignon (1965) qui prennent la relève. Confortés par la déclaration Nostre Aetate (Déclaration sur les relations de l'Église avec les religions non chrétiennes) du Concile Vatican II ${ }^{17}$, ils y organisent, dès 1967, des débats sur des thèmes transversaux (la prière, les pèlerinages, l'hospitalité, etc.), destinés avant tout à un public de croyants et d'initiés, ce qui fait de Vieux-Marché un lieu précurseur du dialogue tel qu'il se développera par la suite en France.

Mais cet élan tend à s'essouffler progressivement. À l'occasion du centenaire de la naissance de Massignon, en 1983, son neveu Jean Cazelles confie ses interrogations relatives à la "survivance " du pèlerinage à Mgr Chaber : "Mais par suite du vieillissement et de la disparition progressive des amis personnels de Louis Massignon, ces aides diminuent. De plus, ces années dernières, la date de ce pardon est tombée en période de Ramadan, de ce fait la participation des musulmans n'a plus été que symbolique. (...) Cette année 1983 (...) peut être un point final ou marquer un nouveau départ ${ }^{18}$. " Il s'agit effectivement d'un tournant décisif : la messe orientale est interrompue, et seuls quelques « massignoniens » continueront de s'y rendre de façon encore plus confidentielle, tandis que le pardon continue comme si de rien n'était.

15. Entretien réalisé en juillet 2008.

16. Lettre de Louis Massignon à Madeleine Grégoire-Doiron (28 octobre 1954) : "J'ai été encouragé, en audience particulière, par Pie XI et par Pie XII, qui m’ont mis parmi les consulteurs officieux de leur commission vaticane (confidentielle) sur les rapports avec l'islam. "

17. Même si l'islam a été l'« invité de la dernière heure au concile ", cf. Avon Dominique, 2005, Les Frères prêcheurs en Orient. Les Dominicains du Caire (années 1910-années 1960), Cerf, Paris, pp. 777-808.

18. Lettre de Jean Cazelles à Mgr Chaber, ancien archevêque de Rabat et nouvel évêque de Perpignan (29 janvier 1983). 


\section{Ambiguitté et hétérogénéité du pèlerinage contemporain}

Tombé en désuétude, le pèlerinage islamo-chrétien des Sept-Saints connaît une seconde vie à partir de 1991, quand un médecin agnostique prend l'initiative, en tant que conseiller municipal, de ranimer la dimension islamo-chrétienne par le dialogue, à la fois interreligieux et interculturel. Pour ce faire, la municipalité de Vieux-Marché créée l'association Sources des Sept Dormants (ASSD) et il est fondamental de souligner que l'initiative émane cette fois d'une volonté politique, laïque, humaniste, et non plus religieuse stricto sensu. En effet, ni l'Association des Amis de Louis Massignon, ni l'Église catholique locale ne sont à l'origine de ce renouveau. Dans une certaine mesure, il s'agit d'une réappropriation locale de l'événement, mais qui ne fait pas l'unanimité comme nous le verrons plus loin.

Qui sont les organisateurs du pèlerinage actuel ? En premier lieu, vient l'Église catholique. Autrefois représentée par le recteur de Vieux-Marché, et aujourd'hui par le prêtre de la paroisse Notre-Dame de Plouaret-Trégor, l'institution religieuse est responsable du pardon catholique des Sept-Saints. C'est avec réserve qu'elle suit, depuis 1954 , le développement du pèlerinage islamo-chrétien, même si elle s'y implique de plus en plus depuis $1999{ }^{19}$.

En second lieu, on trouve les associations locales, indépendantes de l’Église. L'organisation de la dimension islamo-chrétienne revient à l'ASSD depuis 1992. Il s'agit d'une association culturelle qui organise des manifestations (colloques, débats, cérémonies, concerts) pour un public très majoritairement catholique et retraité, mais sans être pour autant liée à l'institution religieuse. En outre, elle n'est pas vraiment bilatérale, ni paritaire car elle ne compte qu'un nombre très réduit de musulmans.

Une autre association, née en 2003, Tud ar Seiz Sant (les «gens des SeptSaints »), a pour but de revitaliser le hameau, et par conséquent le pardon qui s'y déroule. Elle s'occupe principalement de la restauration des pèlerins, et des aspects profanes de la fête (bals, danses, musiques, animations). À ces associations, il faut ajouter celle des Amis de Louis Massignon, domiciliée à Paris, mais dont quelques membres participent chaque année au pèlerinage et aux rencontres, pour que la mémoire de l'orientaliste y soit honorée.

Le public du pèlerinage est particulièrement hétérogène. Nous distinguons principalement les pèlerins du pardon (autochtones et bretons pour la plupart) et les "pèlerins islamo-chrétiens ${ }^{20}$ » intéressés par la rencontre interreligieuse

19. Notamment à la suite de la décisive Conférence des évêques de France tenue à Lourdes, en novembre 1998, sur le thème: "Catholiques et musulmans : un chemin de rencontre et de dialogue ». Cette rencontre a fixé la position de l'Église catholique vis-à-vis de l'islam et a incité les évêques à contribuer dans leurs diocèses au développement du dialogue islamo-chrétien.

20. Nous utilisons cette appellation de «pèlerins islamo-chrétiens » en écho aux "pèlerins de l'interreligieux » décrits par A.-S. Lamine, 2004, p. 103. 
(très majoritairement venus de l'extérieur). À ceux-là, s'ajoutent quelques touristes qui ne participent pas forcément aux rituels de l'un ou l'autre groupe, mais rien ne les empêche de s'y adonner. Ils fréquentent donc le site de façon souvent éphémère et peu approfondie. Notons que leur présence peut être motivée soit par l'aspect traditionnel du pardon, soit par l'aspect insolite de la rencontre islamo-chrétienne, soit par les deux. Un dernier type de visiteurs regroupe ceux qui ont un autre usage du lieu, plus que du pèlerinage. Pour eux, le lieu est sacré, mais pas forcément aux dates du pèlerinage (fin juillet). Ce sont par exemple des adeptes du néo-celtisme et/ou du néo-druidisme, des magnétiseurs ou des " chamanes » issus de la nébuleuse du New Age. Ces derniers sont alors attirés par le dolmen ou la source, beaucoup moins par la chapelle.

Cette hétérogénéité révèle selon nous un "pèlerinage inclusif » au sens où il accueille différents publics et diverses significations. Au fond, de par l'éventail des profils rencontrés, chacun perçoit le sanctuaire à sa manière, tantôt comme un lieu celtique, catholique, islamo-chrétien, patrimonial, touristique, etc. Tout dépend de l'usage et de la lecture que chacun en fait. Or, cela rejoint tout à fait la pensée de John Eade et Michael Sallnow (1991) qui considèrent précisément les pèlerinages comme des «récipients vides" que les pèlerins remplissent du sens de leur choix.

Le dialogue islamo-chrétien, qui est l'une des spécificités de ce lieu de pèlerinage est souvent présenté comme la première œuvre de dialogue islamo-chrétien en France. Chaque samedi après-midi, un colloque interreligieux rassemble plusieurs intervenants (chrétiens et musulmans) devant un public d'environ deux cents personnes. La thématique concerne souvent la question du «vivreensemble » qu'Anne-Sophie Lamine a érigé en nouveau « concept interreligieux » (2004 : 135-170). Remarquons que si la plupart de ces thèmes ont une dimension interreligieuse avérée, il s'en dégage une constante sociale et sociétale. On se préoccupe plus de questions " horizontales » que «verticales ", c'est-à-dire qui touchent plus à la dimension intramondaine qu'à la dimension extramondaine : le vivre-ensemble l'emporte sur la transcendance et l'eschatologie chères à Louis Massignon.

Les intervenants sont généralement des acteurs et des "cadres » du réseau islamo-chrétien qui reviennent souvent d'une année à l'autre. Le public est très majoritairement catholique. Et la forte asymétrie entre chrétiens et musulmans remet en question la portée du dialogue et jusqu'à la raison d'être du pèlerinage. Cette quasi-absence de musulmans traduit un problème de fond majeur, si bien que le partage religieux apparaît tronqué et correspond à ce que Anne-Sophie Lamine appelle : «l'interreligieux (presque) sans les autres »(2005a). 
Dans les faits, l'ethnographie révèle que ce sont les catholiques qui invitent quelques musulmans. L'islam n'est donc qu' " une religion invitée dans un pèlerinage inventé ». Côté musulman, ce sont effectivement toujours les mêmes qui montent à la tribune ou qui sont dans l'assistance : pour la plupart des musulmans libéraux, habitués à ce type de dialogue, et qui viennent de grands centres urbains (Saint-Brieuc, Rennes ou Paris).

Pourtant, les musulmans qui habitent dans les environs de Vieux-Marché ne participent pas au pèlerinage ni aux Rencontres. Une communauté musulmane existe par exemple à Lannion, à quinze kilomètres de Vieux-Marché, où les fidèles se sont constitués en association mais ne se sentent pas concernés par un événement qu'ils perçoivent d'abord comme catholique. En fait, certains y ont déjà participé mais n'y retournent pas. De plus, ils ne sont pas formellement conviés par les associations organisatrices, ce qui traduit un profond dysfonctionnement : on a besoin de musulmans pour justifier le pèlerinage islamochrétien, mais on ne les invite pas directement, si bien qu'ils n'y participent pas d'eux-mêmes. Dès lors, le rapprochement paraît artificiel à bien des égards, et le pèlerinage "islamo-chrétien » en ressort particulièrement fragilisé. L'absence remarquable du Conseil régional du culte musulman (CRCM) de Bretagne vient d'ailleurs confirmer la non prise en compte de l'événement dans le réseau musulman censé représenter l'" islam de France ".

Ce désintérêt musulman se double de celui des autochtones qui ne se sont jamais approprié cette manifestation interreligieuse perçue depuis le début comme un héritage extérieur, imposé " par le haut ", et sans prise directe sur le quotidien. Quelques-uns ont certes essayé d'y assister, mais ils se disent littéralement rebutés par la dimension érudite, voire professorale, de la manifestation. Or ce caractère "intellectuel » fait explicitement partie des projets de l'ASSD depuis sa fondation. Cela conduit à une sorte de boycott de la part des riverains envers des sujets qui n'intéressent qu'un public exogène. La césure n'en est que plus flagrante.

Ajoutons que l'indifférence des locaux tend parfois vers une incompréhension et provoque des élans protectionnistes : "Surtout, ne nous changez pas notre pardon! ", entend-on parfois. Beaucoup éprouvent un sentiment de dépossession et craignent même "que la chapelle ne devienne une mosquée ". En fait, l'islam cristallise certaines tensions suscitées par la peur de l'autre et de l'étranger. En 2008, un habitant du village nous confiait: "Le monde musulman a beaucoup à faire pour aller vers la paix, avec tous les attentats et tout ce qui se passe dans le monde ; alors maintenant, évidemment, on est tout de suite informé de toutes ces choses-là. Ça traumatise un peu notre peuple d'Occident. Il faut essayer d'effacer ces images négatives, mais ce n'est pas facile. Il y a des bonnes paroles, mais il faut que les actes suivent ${ }^{21}$ ! " Derrière l'image d'un lieu d'ouverture et de dialogue, couvent finalement de fortes résistances.

21. Entretien réalisé en juillet 2008. 
L'un des indicateurs les plus marquants concerne "le méchoui de l'amitié » dont il faut relativiser la portée symbolique. Effectivement, depuis quelques années $^{22}$, les moutons utilisés ne sont pas halal ( licites»). Considérée comme secondaire pour certains, cette réalité paraît, en fait, symptomatique de l'état actuel du pèlerinage islamo-chrétien. Certes, on fait un méchoui - à la place du traditionnel cochon grillé - pour rester dans l'esprit du pèlerinage " islamochrétien », mais la contrainte alimentaire islamique sur l'abattage rituel n'est pas respectée. La commensalité en ressort donc amputée. On pense d'abord à satisfaire le public local, sans penser que cela creuse le "fossé » avec les musulmans qui s'estiment pour ainsi dire lésés, pour ne pas dire niés. En fait, derrière les explications de logistique et les normes hygiéniques invoquées par l'association Tud ar Seiz Sant, responsable de la restauration, se profile un obstacle grandissant, et l'on voit comment l'hospitalité affichée se double d'une hostilité latente.

Après avoir souligné ce problème du halal, en 2008, nous pensions que la situation serait arrangée l'année suivante. Mais il n'en fut rien. En 2010, le programme de l'ASSD disait même explicitement : "[Tud ar Seiz Sant] nous invite à partager la tradition culinaire bretonne avec un kik a farz où le porc sera soigneusement séparé des légumes. Le dimanche, ce sera un méchoui mais avec de la viande non halal. Il est toujours possible d'apporter son propre piquenique. » On s'accommode donc d'un problème structurel que personne ne veut régler, sans réaliser que cela fissure la crédibilité de l'événement dit islamochrétien.

Le pèlerinage des Sept Dormants apparaît donc comme un lieu pétri d'ambiguïtés, à commencer par sa dualité structurelle : le pardon d'un côté, et la rencontre islamo-chrétienne de l'autre. Or rien n'est véritablement entrepris pour rapprocher ces deux dimensions juxtaposées : en fait, la petite fête locale se heurte à la vocation universaliste impulsée par Massignon. Au fond, cette hétérotopie dépasse et perturbe les autochtones, ce qui engendre un sentiment de dépossession et d'intrusion touchant parfois à l'islamophobie. Le pèlerinage produit donc aussi des effets opposés à ceux recherchés par ses promoteurs.

\section{* * *}

Depuis quelques années, aussi paradoxal que cela paraisse, un nouveau type de pèlerin est apparu aux Sept-Saints : ceux que nous dirons "séculiers " ou

22. Pourtant, dans les années 1950, Massignon veillait sans cesse à éviter tout dérapage. En 1955, par exemple, il rappelait à l'ordre un de ses amis organisateurs : "J'ai moins apprécié votre propagande à table pour le vin et le porc; cela n'est ni dans la ligne d'un chrétien respectueux des consciences de ses hôtes musulmans (ils étaient nos hôtes...) ", lettre de Louis Massignon à Louis Rouani (7 août 1955). En 1957, il s'indignera à nouveau pour les mêmes raisons : «Il a fallu, encore, qu'à la diffa, on leur propose, en marge du Kouskous et du méchoui, des mets interdits, du vin, du porc ", lettre de Louis Massignon à Louis-Claude Duchesne (août 1957). 
« humanistes ». S'ils sont peu nombreux, ils incarnent cependant une nouvelle tendance : l'ouverture à l'humanisme athée. Or, cela fait polémique et attise une forme de conservatisme de la part des chrétiens attachés à la forme originelle, s'il en est une.

Pleinement lié à la sécularisation de la société française, ce phénomène n'est pas propre à Vieux-Marché et se retrouve dans d'autres lieux de dialogue. Les organisateurs considèrent d'une part que le dialogue ne doit pas se faire à la marge de la société sécularisée, et d'autre part qu'il doit aussi exister un dialogue entre croyants et incroyants. Ainsi, entend-on s'élever des voix issues de la pensée dite " des Lumières ". Le dialogue devient alors « interconvictionnel " (Lamine, 2004 : 259). La croyance est une conviction comme une autre, si bien qu'un incroyant peut participer selon des motivations détachées de toute dimension religieuse. L'idée est de donner la parole à «toutes les personnes de bonne volonté ", afin de rassembler par-delà les clivages politiques et/ou confessionnels, au nom de valeurs citoyennes communes, telles la paix dans le monde, le vivreensemble, la solidarité, etc.

Mais la participation de "libres penseurs " ne reste pas cantonnée au colloque du samedi. Depuis 2008, un athée a initié une minute de silence lors de la célébration à la fontaine à sept veines, "pour une paix sereine », en écho à la formule de Louis Massignon. L'idée est que la paix est universelle, qu'elle n'est pas l'apanage des religions et que les incroyants ont droit à la parole. Présenté comme un moment de recueillement, de prière ou de méditation, selon chacun, ce nouveau rituel constitue assurément une forme de prière sécularisée.

Cette ouverture du dialogue aux incroyants accentue la sécularisation du pèlerinage. La participation de " pèlerins » agnostiques et humanistes, seulement motivés par des préoccupations intramondaines, traduit l' "horizontalisation " du phénomène, c'est-à-dire l'inflexion ou la courbure des intentions verticales et eschatologiques de Massignon. Cela s'illustrant sur place par la volonté des acteurs de développer le dialogue dit « interculturel » au détriment de celui des religions.

En dernier lieu, il faut souligner le développement important des activités « culturelles » pendant le week-end du pèlerinage. L'ASSD organise, par exemple, des expositions, des concerts et des projections de films en marge du colloque qui souffre de sa réputation intellectualiste. L'idée est de rassembler plus de monde autour d'événements plus accessibles et plus grand public. L'association Tud ar Seiz Sant investit encore plus dans ce domaine, en organisant désormais un vide-grenier autour de la chapelle, des « randonnées parlées » censées valoriser le patrimoine des Sept-Saints, des jeux et des bals dansants. La dimension profane a toujours été constitutive des pardons bretons, mais elle connaît ici un fort développement qui atténue la portée spirituelle et résurrectionnelle du message premier des Sept Dormants. 
Pour conclure, on peut dire que le pèlerinage des Sept Dormants est aussi ambigu qu'inattendu. Mais il ne s'agit pas de la même ambiguité des sanctuaires étudiés au début du XX $x^{\mathrm{e}}$ siècle par Frederick Hasluck (2000). C’est ici la notion même de "pèlerinage islamo-chrétien » qui doit être remise en question. En fait, si l'on peut parler à juste titre de pèlerinage au sens large, c'est plus exactement l' « islamo-christianité » qui fait problème dans la mesure où les musulmans sont quasiment absents. Nous sommes d'abord en présence d'un événement catholique, créé par et pour un public catholique soucieux du dialogue. Ainsi, la participation musulmane n'est que symbolique. Pourtant, Louis Massignon souhaitait fonder un lieu de "pèlerinage à égalité » entre chrétiens et musulmans, comme il l'écrivait dès $1954^{23}$.

Mais pour revenir à l'idée de "pèlerinage hétérotopique », nos conclusions sont plus que nuancées dans la mesure où cette hétérotopie islamo-chrétienne ne s'est jamais enracinée localement et qu'elle demeure fragile. Il suffit de constater les tensions et les résistances en présence pour le confirmer. La relation à l'islam est toujours problématique, si bien que le pèlerinage dans son ensemble est toujours subdivisé en deux dimensions : le pardon d'un côté et la "greffe » islamo-chrétienne de l'autre. Bien que communément admise par tous, l'emploi de cette dernière expression empruntée à l'arboriculture est en soi particulièrement signifiante : le volet islamo-chrétien demeure "greffé » et artificiel ${ }^{24}$.

Quoi qu'il en soit, Louis Massignon est apparu comme un «entrepreneur » du religieux œuvrant pour son idéal de réconciliation abrahamique. Il concentre à la fois certaines caractéristiques des modèles wébériens du prêtre et du prophète, si bien qu'il joue de l'un et de l'autre selon les contextes. Il est, en effet, ordonné prêtre depuis quatre ans lorsqu'il crée ce pèlerinage qui représente assurément l'œuvre spirituelle de la fin de sa vie. Depuis lors, le succès de cette entreprise a reposé sur un double charisme : à la fois scientifique (il est professeur au Collège de France) et spirituel (c'est un grand mystique). C'est d'ailleurs ce second aspect qui a été le plus déterminant ; tandis que ses recherches scientifiques ont servi à légitimer son projet spirituel.

Cet article contribue au champ d'étude récemment ouvert des pèlerinages partagés par plusieurs religions ou confessions (Albera, Couroucli, 2009). Mais ce cas d'étude s'avère profondément différent des situations observées dans le

23. Lettre de Louis Massignon au Pr. Hamidullah (15 juin 1954).

24. On parle volontiers de "greffe " aujourd'hui, même si Louis Massignon réfutait cette appellation : "l'Evêché de St Brieuc (...) reste complètement inerte depuis 10 ans (...) car il voit dans ce pèlerinage une "greffe" grecque sur un pèlerinage breton latin traditionnel " [souligné dans l'original], lettre de Louis Massignon à André Holleaux, directeur de cabinet d'André Malraux (16 août 1962). Le caractère "grec » de la greffe est dû à la messe en rite melchite que Massignon institua dès 1955. 
monde méditerranéen, dans la mesure où il s'agit d'un pèlerinage organisé et pensé comme tel: la mixité islamo-chrétienne n'y est pas spontanée comme ailleurs, mais s'est avérée artificielle et intellectualisée "par le haut ». Les "pèlerins islamo-chrétiens " n'invoquent pas particulièrement les saints, mais participent au rassemblement pour le dialogue interreligieux. Cela représente donc une autre facette du phénomène de la mixité religieuse, voire une nouvelle forme de mixité intellectualisée dans un contexte sécularisé.

Au regard de la situation contemporaine, ce lieu de pèlerinage a été " déverrouillé " par Louis Massignon, ce qui a engendré une succession d'initiatives et d'agrégations que l'orientaliste était loin d'imaginer. Cela se traduit dans les faits par une forte hétérogénéité des pèlerins, des attentes et des pratiques ${ }^{25}$. Et contrairement à la vision turnerienne de la communitas, le site des Sept-Saints ressort comme un espace de tensions et de concurrence entre les différents " usagers ». Plutôt qu'un espace-temps où les statuts sociaux seraient estompés, les identités respectives sont renforcées, voire exacerbées. Cette hétérogénéité est avant tout matricielle de discours, de représentations et de différences, ce qui rejoint la pensée de John Eade et Michael Sallnow sur les pèlerinages comme "arènes » et lieux de conflit (1991). Or, cela est particulièrement paradoxal pour un pèlerinage de dialogue et de réconciliation.

Quid des Sept Dormants ? Ils ont été « réveillés » par Massignon parce qu'ils incarnaient, selon lui, le témoignage de la résurrection de la chair. Ils ont donc servi de «trait d'union » islamo-chrétien. Cependant, au pèlerinage aujourd'hui, le message principal associé aux Dormants n'est plus la résurrection mais le dialogue et le vivre-ensemble. Les Dormants ne sont plus des médiateurs entre Dieu et les hommes, mais entre les hommes dans un sens horizontal. Ils sont donc devenus le symbole d'une rencontre possible entre chrétiens et musulmans, ainsi que d'un dialogue qui demeure avant tout symbolique. Certes, on se réunit sous le signe des Sept Dormants, mais ces derniers ont tendance à être plus ou moins oubliés...

Force est donc de constater une indéniable courbure du projet massignonien, qui se traduit notamment dans la sécularisation du phénomène. Certes, il paraît bien fragile par certains aspects. Mais malgré les difficultés rencontrées depuis sa fondation, le pèlerinage des Sept Dormants s'invente et se réinvente sans cesse selon les défis du présent, ce qui constitue une preuve certaine de vitalité. Sans pouvoir présumer de son avenir, il semble que l'Église catholique soit en train de le "récupérer » pour mieux l'institutionnaliser, mais n'était-ce pas au fond ce que voulait Louis Massignon?

25. Nous n'avons malheureusement pas pu développer ici le cas de l'occupation néo-celtique qui revendique sa légitimité par l'antériorité des cultes pratiqués dans le dolmen. 
En définitive, le sujet de cette monographie dépasse la seule Bretagne et concerne la société entière. Il pose la question de la pluralité religieuse, du vivreensemble et du dialogue des religions dans un monde sécularisé. À l'heure où l'islam en France occupe à nouveau le devant de la scène politique et médiatique, ce pèlerinage islamo-chrétien n'apparaît-il pas comme un "laboratoire » de ce qui se passe à plus grande échelle ? L'idée d'expérimentation interreligieuse permet de rendre compte que ce pèlerinage révèle et reflète, dans un certain sens, quelques facettes de la société contemporaine dont il serait le miroir. L'un des enjeux de base étant : comment cohabiter dans un environnement pluraliste et comment se comporter avec l'autre religieux et/ou culturel ? La métaphore de Michel Foucault sur le miroir hétérotopique s'avère dès lors particulièrement instructive :

« Je crois qu'entre les utopies et ces emplacements absolument autres, ces hétérotopies, il y aurait sans doute une sorte d'expérience mixte, mitoyenne, qui serait le miroir. Le miroir, après tout, c'est une utopie, puisque c'est un lieu sans lieu. (...) Mais c'est également une hétérotopie, dans la mesure où le miroir existe réellement, et où il $\mathrm{a}$, sur la place que j'occupe, une sorte d'effet en retour ; c'est à partir du miroir que je me découvre absent à la place où je suis puisque je me vois là-bas. (...) le miroir fonctionne comme une hétérotopie en ce sens qu'il rend cette place que j'occupe au moment où je me regarde dans la glace, à la fois absolument réelle, en liaison avec tout l'espace qui l'entoure, et absolument irréelle, puisqu'elle est obligée, pour être perçue, de passer par ce point virtuel qui est là-bas. » (Foucault, 2001 : 1575)

Focaliser sur ces effets et jeux de miroir (là-bas) permet donc bien de réaliser autre chose (ici). Le pèlerinage serait l'occasion de percevoir et de grossir certains aspects moins perceptibles à l'œil nu. Sa caractéristique d'être un lieu circonscrit permet de mieux observer certains détails qui traversent le reste de la société. Reste à tenir compte du fait qu'un miroir peut être déformant, grossissant, mal poli ou ébréché. Il peut également prendre la forme d'une boule à facettes, ce qui renvoie aussitôt à l'idée d'une réalité complexe et mosaïque.

Enfin, pour rester proche de la pensée de Foucault, il faudra développer cette notion de "pèlerinage hétérotopique ". Dès 1967, le philosophe appelait justement à la nécessité d'une approche qu'il appelait " hétérotopologie » : non pas une science à proprement parler, mais une « description systématique qui aurait pour objet, dans une société donnée, l'étude, l'analyse (...) de ces espaces différents, ces autres lieux, une espèce de contestation à la fois mythique et réelle de l'espace où nous vivons" (Foucault, 2001 : 1575). Dans une certaine mesure, cette étude espère contribuer à ce champ hétérotopologique toujours en devenir.

Manoël PÉNICAUD

IDEMEC-Maison Méditerranéenne des Sciences de l'Homme manoelpenicaud@gmail.com 


\section{Bibliographie}

Albera Dionigi, Couroucli Maria, (dir.), 2009, Religions Traversées, Arles, Actes Sud.

Avon Dominique, 2005, Les Frères prêcheurs en Orient. Les Dominicains du Caire (années 1910-années 1960), Paris, Éditions du Cerf.

BADONE Ellen, 2007, "Echoes from Kerizinen: Pilgrimage, narrative, and the construction of sacred history at a Marian shrine in Nortwest France ", Journal of the Royal Anthropological Institute, (NS)-13, pp. 453-470.

BleuZEn Brigitte, 2010, "Du Tro-Breiz à la Vallée des Mille Saints », Archives de Sciences Sociales des Religions, 151, pp. 229-249.

Eade John, Sallnow Michael, (dir.), 1991, Contesting the Sacred. The Anthropology of Christian Pilgrimage, Londres-New York, Routledge.

Foucault Michel, [1994] 2001, "Des espaces autres ", Dits et écrits, Paris, Gallimard, t. II, pp. 1571-1581.

HasLuCK Frederick, [1929] 2000, Christianity and Islam under the Sultan, Istanbul, The Isis Press.

JouRDAN François, [1983] 2001, La tradition des Sept Dormants. Une rencontre entre chrétiens et musulmans, Paris, Maisonneuve et Larose.

LACOMbe Philippe, 2000, "Corps, culture, religion : la dimension profane des pardons bretons ", Ethnologie Française, XXX-1, pp. 109-118.

Lamine Anne-Sophie, 2004, La cohabitation des dieux. Pluralité religieuse et laïcité, Paris, Presses Universitaires de France, coll. "Le lien social ".

-, 2005a, «Les relations islamo-chrétiennes à l'épreuve des générations », Social Compass, 52-2, pp. 131-142.

-, 2005b, "Mise en scène de la "bonne entente" interreligieuse et reconnaissance ", Archives de sciences sociales des religions, 129, pp. 83-96.

Laurent Donatien, 2004, "La Gwerz des Sept Saints Dormants ", Hopala! La Bretagne au monde, $\mathrm{n}^{\circ} 17$, pp. 32-42.

L'YvonnEt François, 1999, "La géographie spirituelle de Louis Massignon ", Bulletin de l'association des Amis de Louis Massignon, 9, pp. 15-27.

MASSIGNON Louis, 1954-1963, "Les Sept Dormants d'Éphèse (ahl Al-Kahf) en Islam et en Chrétienté. Recueil documentaire et iconographique ", Revue des Études Islamiques, Paris, Geuthner, huit parties.

-, 1992 (1958), "La crypte-dolmen des VII Saints Dormants d'Éphèse ", Extrait des Mémoires de la Société d'Émulation des Côtes-du-Nord, Saint-Brieuc, Les Presses Bretonnes.

-, 2009a, Écrits Mémorables, tomes 1-2, textes établis, présentés et annotés sous la dir. de Jambet C., par Angelier F., L'Yvonnet F., et Ayada S., Paris, Robert Laffont, coll. «Bouquins ».

-, [1950] 2009b, «Les Sept Dormants, Apocalypse de l'Islam », in Massignon L., Écrits Mémorables, t. I, pp. 321-335.

Morinis Alan, (dir.), 1992, Sacred Journeys. The Anthropology of Pilgrimage, Westport, Greenwood Press.

Moubarac Youakim, 1961, Le culte liturgique et populaire des VII Dormants Martyrs d'Éphèse (Ahl al Kahf). Trait d'union Orient-Occident entre l'Islam et la Chrétienté, Rome, Pontificia Univesitas Gregoriana. 
PÉNICAUd Manoël, 2011 (à paraître), «Un projet de réconciliation islamo-chrétienne en Méditerranée sous le signe des Sept Dormants (1950-1962) », in Albera D., Crivello M. et Tozy M., La réinvention du "lien» en Méditerranée, Aix-en-Provence, Presses Universitaires de Provence.

PIETTE Albert, 2000, La religion de près. L'activité religieuse en train de se faire, Paris, Métailié.

RenAn Ernest, 1878, "La légende des Sept Dormants en Basse Bretagne », in Gaidoz H., Rolland E., (dirs.), Mélusine, Paris, col. 204-205.

SAIID Edward, [1978] 1980, L'Orientalisme. L'Orient créé par l'Occident, Paris, Seuil.

Trévidy Jean, 1897, "Les Sept Saints de Bretagne et leur pèlerinage ", Bulletin archéologique de l'Association Bretonne, Congrès de Rennes.

Turner Victor, Turner Édith, 1978, Image and Pilgrimage in Christian Culture: Anthropological perspectives, Oxford, Basil Blackwell.

Weber Max, 1996, Sociologie des Religions, Paris, Gallimard.

\section{Résumé}

Le pèlerinage des Sept Dormants est aujourd'hui considéré comme l'une des plus anciennes ouvres de dialogue islamo-chrétien en France. Dès 1954, Louis Massignon inaugurait cette rencontre en la "greffant " sur un "pardon" breton dédié aux Sept Dormants d'Éphèse, également connus en islam sous le nom de Abl al-Kahf. Or, cette entreprise ressort comme une "hétérotopie ", c'est-à-dire une utopie localisée dans l'espace. Toujours actif à ce jour, le pèlerinage est désormais doublé de rencontres interreligieuses et interculturelles. L'ethnographie fait apparaître une nouvelle facette du phénomène des lieux saints partagés par plusieurs confessions. Il s'agit en effet d'un pèlerinage pensé pour le dialogue et où la mixité n'est pas spontanée. Tout en promouvant le dialogue, le pèlerinage apparaît aussi comme un lieu de concurrence et de tensions, y compris envers l'islam qui s'avère n'être qu'une religion invitée dans le cadre d'un événement catholique.

Mots-clés : pèlerinage, dialogue islamo-chrétien, hétérotopie, Sept Dormants, Louis Massignon.

\section{Abstract}

The Seven Sleepers Pilgrimage is nowadays considered to be one of the oldest enterprises of Christian-Muslim dialogue in France. As early as 1954, Louis Massignon initiated this gathering by "grafting" it on a Breton pardon (pilgrimage) dedicated to the Seven Sleepers of Ephesus, also known in Islam as Abl al-Kahf. In fact, this initiative is an "heterotopia", ie an utopia localized in space. Still active today, the pilgrimage is now combined with inter-religious and intercultural conferences. The ethnography reveals a new aspect of the phenomenon of shrines shared by several religions. Indeed, this pilgrimage is "invented" and "thought" for dialogue, so that mixity is not spontaneous. While promoting dialogue, the pilgrimage became also as a place of competition and tension, including towards Islam which turned out to be a religion invited to a Catholic event.

Key words: pilgrimage, christian-muslim dialogue, heterotopia, Seven Sleepers, Louis Massignon. 


\section{Resumen}

La peregrinación de los Siete Durmientes es hoy considerada como una de las obras más antiguas de diálogo islámico-cristiano en Francia. Desde 1954, Louis Massignon iniciaba este encuentro "injertándolo" sobre un "perdón" bretón dedicado a los Siete Durmientes de Éfeso, igualmente conocidos en el Islam con el nombre de Abl al-Kahf. Ahora bien, esta empresa resurge como una "heterotopía", es decir, una utopía localizada en el espacio. Aún activa, la peregrinación es doblada por encuentros interreligiosos e interculturales. La etnografía hace aparecer una nueva faceta del fenómeno de los lugares santos compartidos por varias confesiones. Se trata en efecto de una peregrinación pensada para el diálogo y donde la mixtura no es espontánea. Promoviendo siempre el diálogo, la peregrinación aparece también como un lugar de competencias y de tensiones, también hacia el Islam que comprueba que es sólo una religión invitada a un acontecimiento católico.

Palabras clave: Peregrinación, diálogo islámico-cristiano, heterotopía, Siete Durmientes, Luis Massignon. 